\title{
$\therefore$ Detection of Heart Problem Using Internet of Things Application
}

\section{IJCRR}

Section: Healthcare

ISI Impact Factor

(2019-20): 1.628

IC Value (2019): 90.81

$\operatorname{SJIF}(2020)=7.893$

(c) (i) (3)

Copyright@IJCRR

\section{Sesha Vidhya S', Shanthi KG'ㅐ, Manikandan A², Senthil Kumar $K^{3}$}

'Department of ECE, RMK College of Engineering and Technology, Tamil Nadu, lndia; 'Department of ECE, Amrita School of Engineering, Amrita Vishwa Vidyapeetham, Chennai, Tamil Nadu, India; ${ }^{3}$ Department of ECE, Rajalakshmi lnstitute of Technology, Chennai, Tamil Nadu, India.

\section{ABSTRACT}

Introduction: According to World Health Organisation (WHO), Heart Disease is the leading cause of Population Death. The World Health Organization survey says that heart disease is the common cause of population death in the world. The heart's main function is to pump blood into the circulatory system of human beings; if one of its ventricles fails to work, the heart gets attacked, and in due course, leads to death if not resuscitated on time. Most of the time, a heart attack results in abrupt death before the patients get any consideration from a medical professional.

Objective: This paper proposes an Electro Cardiogram (ECG) monitoring system which detects the heart problem using loT (Internet of Things) applications.

Methods: It is about the IoT Based ECG Monitoring with AD8232 ECG Sensor and ESP-32 developing kit using an online loT platform called Ubidots, which captures the sensor data and turn it into useful information. It also measures an ECG signal and transmits it to a smartphone via WiFi for data analysis.

Results: The ECG signal from a patient is viewed by the doctor using their smartphone. The system utilizes the user's smartphone for data processing, and the built-in communication is used to generate an ECG waveform. By analysing the ECG waveform, it detects the heart problem, if any. If the heartbeat rate is under abnormal condition, then the Ubidots platform sends a message to the doctor stating that the patient condition is abnormal. At the same time, the Buzzer and Light Emitting Diode (LED) also make an alert to notify the condition of the patient of the caretaker.

Conclusion: Since the last few decades, heart diseases are becoming a greater issue and many people lost their lives because of such health problems. Hence, heart disease cannot be taken lightly. By analyzing and continuously monitoring the ECG signal at the initial stage, heart disease would be prevented.

Key Words: WHO (World Health Organization), ECG (Electro Cardio Gram), loT (Internet of Things), WBAN (Wireless Body Area Network), GSM (Global System for Mobile Communications), (SMS) Short Message Service.

\section{INTRODUCTION}

The World Health Organisation survey says that the heart disease is the common cause of population death in the world. The heart's main function is to pump blood into the circulatory system of human beings; if one of its ventricles fails to work, the heart gets attacked, and in due course, leads to death if not resuscitated on time. Most of the time, a heart attack results in abrupt death before the patients get any consideration from a medical Professional. For instance, in the republic of China, they used to practice a traditional health care system. This means that the concerned patients will make a call to the healthcare service centres themselves.
In case of sudden heart attack, the unconscious patient may not be able to call the service, and hence the unwanted death situation will occur. With the help of IoT technology, the heart attack problem is overawed.

The patients now switch the system from inactive to a pervasive one and trigger the health care services to be aware of his/her situation. Hence, this calls for a remote patient monitoring system. ${ }^{1}$ The IoT plays an essential role in our day to day routine activities. It provides the means through which many machines and devices are monitored and controlled remotely without human intervention. ${ }^{2}$ The IoT technology tremendously increases the data value, performance,

\section{Corresponding Author:}

Ms. S. Sesha Vidhya, Associate Professor, RMK College of Engineering and Technology, Tamil Nadu, India. E-mail: seshavidhya@rmkcet.ac.in

ISSN: $2231-2196$ (Print)

Received: 17.09 .2020
ISSN: 0975-5241 (Online)

Revised: 10.11 .2020
Accepted: 21.12 .2020
Published: 07.05 .2021 
efficiency, accuracy, speed of the system operation and output results. The advancement in the IoT technology coupled with the sensors has brought tremendous development in the health care system. Sensors along with an integrated IoT health care system has provided the possibilities of monitoring and control of patient's data from anywhere in the world. There is the need to have medical personnel close to the patient for direct monitoring and control for better monitoring of the patient. ${ }^{3}$ For the past few years flourishing attention is incorporated in the field of Biomedical communication for the advancement of a one-to-one care system to perceive greatly a hominoid dynamic body part action. Wireless body area network (WBAN) is used for measuring an Electrocardiogram (ECG) signal and transmitting it to a smartphone via WiFi for data analysis. ${ }^{4}$

However, having medical personnel to take care of the patient at all times is impossible due to their scarcity and tight schedules. Therefore, remote patient monitoring and control is the best proposal to take appropriate medical care of the patient. This kind of approach provides physical parameters that are required by the medical personnel, and the data is transmitted remotely via the internet. Hence, the dedicated approach for monitoring heart activity and controlling its parameters is essential.

\section{LITERATURE REVIEW}

In recent years wireless body area network and the Internet of things have strongly emerged in the medical field for the health care of the patient. It collects the patient's data regarding the heartbeat rate and pulse rate. The collected data is continuously monitored and will be sent to a Doctor.

Vaishnave et al, proposed the measurement of the human heartbeat and body temperature using Arduino UNO and sends the information to clients end utilizing microcontroller with sensible cost. ${ }^{5}$ Patel et al., presented detection of Heart attack and heart rate monitoring using IoT. ${ }^{6}$ It consists of an android WIFI module, a heartbeat sensor and a pulse sensor. The heart rate readings have been sensed by the pulse sensor and the result is displayed on the LCD (Liquid Crystal Display) screen. They have used a set-pointer through the system which helps in determining whether a person is healthy or not, by checking their heartbeat and comparing it with the set point. If the setpoint is beyond the limit the system will send an alert message. But there is a chance to display the false result due to varying limits in set-pointer.

Gurjar et al. proposed Heart attack recognition by heartbeat identifying using IoT. ${ }^{7}$ The components used in this work is ATMEGA 328, heartbeat sensor, temperature sensor, and pressure sensor. The transmitter circuit includes AVR (Alf and Vegard's RISC (Reduced-instruction-set Computing)) family microcontroller interfaced to the LCD screen to dis- play, the receiver circuit includes an LED device and Buzzer sound which is used to alert a person. The main drawback of this method is, it alerts the patient only. Later the patient has to go and consult a Doctor.

Ajitha et al. depicted IoT based heart attack finding and alert system. ${ }^{8}$ The tools used in this work are an AVR controller, wireless module, Analog sensor, ECG leads. This project uses an alert and LCD that are capable of monitoring the heart rate. But there is no message alert to the Doctor. Barai et al. dealt with the comparison of non-invasive heart rate monitoring system using GSM module and RF module. Internet of things IoT based heart attack and heart rate measurement using Arduino is promising for remote monitoring of heart rate, yet it does not offer any real-time monitoring system. ${ }^{9}$

Gupta et al. proposed a smart Health Care Kit based on IoT. ${ }^{10}$ Their system collects data such as Heart rate, blood pressure and ECG signal of the patient and sends alerts to the doctor so that he has full knowledge of the patient's health from time to time. But their system lacks in collecting diabetic conditions of the patient and, therefore, would be incapable to detect heart attack caused by diabetes or obesity. Mamidi et al. proposed an IOT based heart attack detection and heart rate monitoring. The components used are RFID or near field communication technology. At first, they record the default values of pulse rate, systolic pressure so that the application records the user's activity and check the collected data with the default values whether it is near to it or not. The system which they proposed has the quality of detecting heart attacks with the help of monitoring heart rate and blood pressure based on IoT. But in this method, there is a possibility of hacking the data.

Mallick et al. presented a heart rate monitoring system using fingertip through Arduino and processing software. ${ }^{11}$ This technology uses NodeMCU (Lua based firmware for the ESP8266) and fingertip sensor. It is based on the principle of Photo Plethysmography (PPG) which is a non-invasive method of measuring the variation in blood volume in tissue using a light source and detector. The heart rate counting and monitoring are performed by the processing unit. But the fingerprint sensor does not take into consideration when a person's finger changes in sizes or form/pattern over time. Raihan et al., portrayed Smartphone-based ischemic heart disease risk prediction using clinical data and data mining approach. ${ }^{12}$ The tools used in this are chi. square, ACS, IHD, Data mining. The main aim of this work is to make a simple approach to detect the risk of IHD and to avoid sudden death. The main drawback is that there is no alerting system to alert the patient.

Prittopaul et al. approached the cyber-physical system for the detection of heart attack using wireless monitoring and actuation system. ${ }^{13}$ This involves a Cyber-Physical System 
(CPS) which contains a small wearable device to transmit the ECG signal to the patient's mobile phone. In case of any emergency or irregularities in the heartbeat, it makes an alert. But, this model only detects heart failure that occurs due to variation in heart rate as the system is completely based on the data gathered by ECG signals and neglects the heart failure that is caused due to smoking, obesity, alcohol intake, physical inactivity etc.

Pawar et al. proposed a system for heart rate monitoring using Arduino Uno and an IR based sensor. ${ }^{14}$ It is used to monitor the physical parameter like heartbeat and then it sends the data that is measured, to a doctor through a short message service. The system also consists of an IR base heartbeat sensor, GSM module and an Arduino UNO. But IR sensors can't measure an accurate value for heart rate. Das et al presented a Microcontroller based low-cost heart rate counter. ${ }^{15}$ The Microcontrollers have been used to measure heart rate using an Infrared (IR) sensor but IR sensors can't measure an accurate value for heart rate and are not versatile too.

Ashrafuzzaman, et al. depicted Heart attack detection using a smartphone. ${ }^{16}$ The technology is used in this paper are data mining, fuzzy logic. It detects the heart attack by placing the index finger on a mobile camera to determine the peak value of blood pressure. The main idea of the proposed system is to find out the average distance between adjacent peaks for heart rate calculation. Issac et al. presented a real-time heart monitoring system using an android application. ${ }^{17}$ The technology used in this paper are CUEDETA (A Real-Time Heart Monitoring System Using Android Smartphone) and also contains a location tracking ECG sensor. This paper is used to monitor the ECG signals of the patient and to send alerts to his/her contacts. The alert message has been used to find the exact position of the patient through google map. But there is a chance to detect false alerts due to arranged mode.

Srinivas et al. described an analysis of coronary heart disease and prediction of a heart attack in coal mining regions using a data mining technique. ${ }^{18}$ This paper involves various algorithms for the prediction of a heart attack. The technologies used in this paper are, Decision Tree, Neural Network, Multilayer Perceptron (MLP), Bayesian model and SVM with an accuracy of $82.5 \%, 89.7 \%, 82 \%$ and $82.5 \%$ respectively. According to their result, Multilayer Perceptron acquires the highest accuracy of $89.7 \%$ as compared to other algorithms since the syndrome is a collection of symptoms, and it is a concept which is developed employing mapping symptoms to Traditional Chinese Medicine (TCM) expert's brain. As the syndrome is recognized by the human brain, multilayer perceptron is considered a good model. According to their research, it may implement false alarm rate because of time series modelling and more continuous data should be used instead of categorical data to study patient's morbidity condition concerning clinical care.
Landaeta, et al, portrayed the detection of heart rate from plantar bioimpedance measurements. ${ }^{19}$ They have proposed a technique for measuring heart rate and it also relies on electrical impedance variations which are detected by a plantar interface with both feet, such as those in some bathroom weighing scales for the analysis of body composition. The Heart-related impedance variations that arise in the legs come from arterial blood circulation and are below $500 \mathrm{~m}$ Omega. But usage of feet makes it uncomfortable for many patients.

\section{PROPOSED WORK}

We proposed a system, which consists of an ECG sensor that uses electrodes connected to the ECG board via standard electrode cables to measure an ECG signal from the patient. The measurements have been sent to the ESP32 controller, where they are processed and sent to the IoT Cloud Platform. Then, the data has been broadcast to the user's phone via WIFI. To publish the data to the IoT Cloud, we need some IoT platform. So Ubidots is one such platform that offers a platform for developers that allows them to catch the sensor data and turn it into a piece of useful information. The Ubidots platform is used to send data to the cloud from any Internet-enabled device which will turn the sensor data into information that matters for business decisions, machine-to-machine interactions, educational research, and thereby increase the economization of global resources. It serves as an easy and affordable means to integrate the power of the IoT into your business/research. Its platform for application enablement will support interactive, real-time data visualization (i.e. widgets), and also an IoT app builder that provides developers to extend the platform with their HTML code for individualization. Ubidots empower the data from device to visualization.

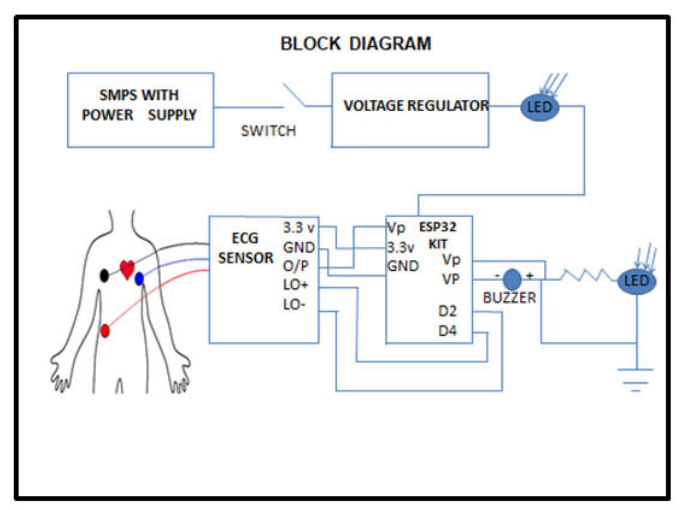

Figure 1: Block Diagram.

Figure 1 describes the working principle of the proposed work. The ESP32controller is connected to the AD8232ECG sensor. Remaining all of the components and the electrodes, are placed in an assembly box. The switched-mode power 
supply (SMPS) of $12 \mathrm{v}$ is given to the voltage regulator as it will provide only the required voltage to the ESP32 controller. The ESP32 controller will operate at a voltage of $3.3 \mathrm{v}$. The electrodes are placed on the chest of the patient and hence the heart beats are obtained which will be of analog form. So, for achieving efficient transmission of data, it is converted into digital form using AD8232 ECG sensor. Then the digital data obtained is given to the ESP32 controllerwhich acts on the signal, based on the instructions that are coded in embedded C language. Then the signals are interfaced with the Ubidots, an IOT platform to view the ECG signal. By analysing the ECG waveform, it detects the heart problem, if any. If the heartbeat rate is beyond a certain limit (abnormal condition), then the Ubidots platform sends an SMS message to the Doctor, stating that the patient condition is abnormal. At the same time, the Buzzer and LED also make an alert to notify the condition of the patient of their caretaker.

\section{ECG Sensor}

The ECG sensor used is a low-cost board, used to measure the electrical activity of the heart. This electrical activity of the heart is viewed as ECG or Electrocardiogram and output as an analogue reading. ECG signal what we obtained is noisy, therefore used AD8232 an Op-amp to obtain a clear signal from the PR, QT and ST intervals easily. The AD8232 acts as signal conditioning for ECG and it is also used to measure other bio-potential measurement signals. It is designed to amplify the bio-potential signal in the presence of noise.

\section{ESP- 32 Developing Kit}

The AD8232 Op-amp consists of nine connection pins and wires. The other connectors include $\mathrm{LO}+$, LO-, OUTPUT, 3.3V, GND are the essential pins used for operating the Opamp with an Arduino. Also, three lead electrodes on this board are provided, RA (Right Arm), LA (Left Arm), and RL (Right Leg). The electrodes are placed at the particular location of the body to obtain an ECG signal because only at the particular location, the required frequency of the heart is obtained.

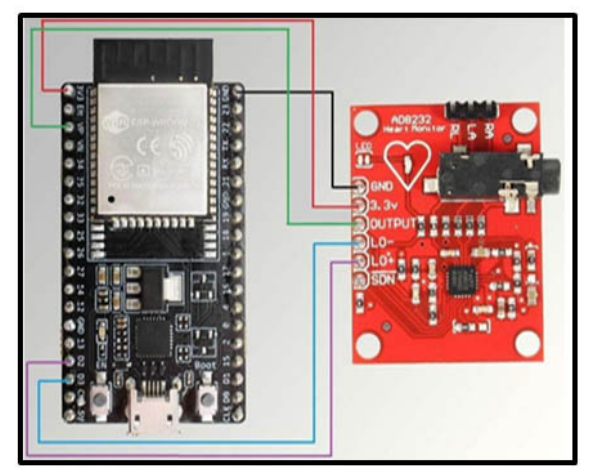

Figure 2: Interfacing AD8232 ECG Sensor With ESP32.
Figure 2 represents the interfacing AD8232 ECG Sensor with ESP32-WROOM-32 developing kit. The AD8232 is power supplied with $3.3 \mathrm{~V}$ from the ESP32 module. The output pin of AD8232 will be an analogue signal. This pin is then connected to the VP pin of ESP32-WROOM-32. Similarly, LO and $\mathrm{LO}+$ ofAD8232 are also connected to the pin D3 and D2, respectively on ESP32-WROOM-32.

\section{SYSTEM DESIGN AND IMPLEMENTATION}

This paper presents the design and implementation of a human heartbeat rate monitoring using a heart pulse sensor and IoT-based technology. This sensor senses the human heartbeat and is recorded. The read data are processed by the ESP32 controller and transmitted to the WiFi module for uploading to the internet server platform (Ubidots) for further analytics and visualization. When the data was captured the same is processed and stored in real-time with a date and time stamp. The proposed work consists of the ECG sensor unit, the power supply unit and the user interface unit as the input units. The WiFi Module unit serves as the output unit. The ESP32 controller unit monitors and controls the signals. The system is programmed using embedded $\mathrm{C}$ programming language.

The ECG sensor will sense the heartbeat. The sensed data from the sensor is transmitted to the analogue to a digital converter (ADC) for conversion to a digital signal. The obtained digital signal is then transmitted to the ESP32 controller. The ESP32 controller acts on the signal based on the instructions which are coded in embedded $C$ language. Furthermore, the data are sent in real-time to the WiFi module and transmitted to the webserver (Ubidots)for further analytics and visualization. The analyzed data are updated synchronously in real-time to display the status of the human heartbeat rate. It is also associated with a Buzzer and an LED, which will be active when the heart condition goes abnormal. An SMS alert will also be sent to the Doctors.

\section{Android Application}

The Android application is made to handle data from the sensor. The application has two modes: one that displays ECG voltage readings and another that shows the SMS alert to the Doctor. As the application is launched, it performs a scan for devices and allows the user to select the ECG sensor. The application then receives and interprets data from the sensor and displays it on the screen as a plot. It involves the implementation of an IoT based ECG Monitoring with AD8232 ECG Sensor and ESP32 developing kit using an online IoT platform called Ubidots. The ECG sensor AD8232 has been interfaced with ESP32, then it generates an ECG signal by connecting ECG electrode in the chest of the patient.The ECG signal from a patient heart has been viewed continu- 
ously by the Doctor using their smart phone. Using parameters of Ubidots, the ECG graph will be sent to cloud. The experimental results that are obtained from the implemented prototype were found to be accurate. Because the system is up to sense the data and then through the internet it transmits the data that has been sensed. Since the last few decades, heart diseases are becoming a greater issue and many people lost their lives because of such health problems. Hence, the heart disease cannot be taken lightly. By analyzing and continuously monitoring the ECG signal at the initial stage, heart disease would be prevented (Figure 3).

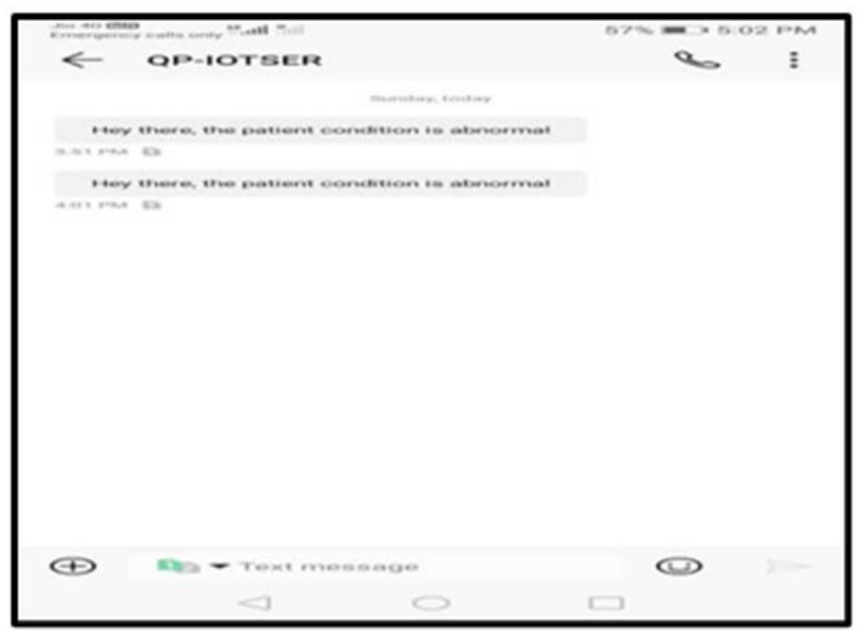

Figure 3: SMS Alert Message.

The Short Message Service (SMS) allows text messages to be sent and received from mobile phones. An alert message stating that 'the patient condition is abnormal' will also be sent to all the Doctors available, in case of any emergency of abnormal conditions made. This is accomplished by GSM (Global System for Mobile communications) mechanism.

\section{Conclusion and Future Work}

We proposed an IoT based heartbeat monitoring with AD8232 ECG Sensor and ESP32 developing kit. Since the last few decades, heart diseases are becoming a greater issue and many people lost their lives because of such health problems. Hence, heart disease cannot be taken lightly. By analyzing and continuously monitoring the ECG signal at the initial stage, heart disease would be prevented. The ECG signal obtained is discussed and reviewed. The IoT based computational frameworks for heart monitoring play a crucial role in early diagnosis, prediction and cardiac disease management. This experimental work explores an IoT connected low power wireless sensor interface implementation for long term monitoring of cardiac parameters. The regular use of the device is very helpful for preliminary detection of heart diseases and to reduce severe damage and mortality rate due to cardiovascular diseases. Similar to this user-friendly ECG monitoring system, additional health monitoring systems such as temperature measurement, Blood Pressure, Diabetes, etc., can be developed using IoT that will greatly help to decrease existing health problems to a certain amount.

\section{ACKNOWLEDGEMENT}

Authors acknowledge the immense help received from the scholars whose articles are cited and included in references of this manuscript. The authors are also grateful to authors/ editors/publishers of all those articles, journals and books from where the literature for this article has been reviewed and discussed.

\section{Conflict of Interest: NIL}

\section{Source of Funding: NIL}

\section{REFERENCES}

1. World Health Organization. The top 10 causes of death. [Online]. 2014. Available:http://www.who.int/mediacentre/factsheets/ fs310/en/

2. Mehmet T. IoT Based Wearable Smart Health Monitoring System. Celal Bayar Uni J Sci 2018;14(3).

3. Mohammad SI, Mohammad TI, Ali FA, Gan KB, Norbahiah M, Amin N. Monitoring of the Human Body Signal through the Internet of Things (IoT) Based LoRa Wireless Network System. Applied Science 2019;9(9).

4. Wolgast G, Helander J, Johansson E, Ehrenborg C, Israelsson A, Manefjord H. Wireless Body Area Network For Heart Attack Detection. IEEE Anten Propag Mag 2016;58(5).

5. Vaishnave AK, Jenisha ST, Tamil Selvi S. IoT Based Heart Attack Detection, Heart Rate and Temperature Monitor. Int Res J Multidisc Tech Innov 2019;02.

6. Patel N, Patel N and Patel P. Heart Attack Detection and Heart Rate Monitoring using IOT. Int J Innov Adv Comp Sci 2018;7(4).

7. Gurjar AA, Sarnaik NA. Heart Attack Detection By Heartbeat Sensing using Internet of Things. Int Res J Engi Technol 2018;5(3).

8. Ajitha U, Aswathi PA, Sasidaran A, Muhammed VA, Anand V, Arvind A. IOT Based Heart Attack Detection and Alert System. Int J Engi Manag Res 2017;7(2).

9. Barai AR, Rahman MR, Sarkar AK. Comparison of non-invasive heart rate monitoring system using GSM module and RF module. 2nd International Conference of Electrical \& Electronics Engineering (ICEEE), Rajshahi 2017.

10. Gupta P, Dhirand PK. IoT Based Smart Healthcare Kit. International Conference on Computational Techniques in Information and Communication Technologies 2 (ICCTICT), IEEE, 2016; 237-242.

11. Mallick B and Patro AK, Heart Rate Monitoring System Using Fingertip through Arduino. Int J Sci Engi Technol Res 2016;5(1).

12. Raihan M, Saikat Mondal. Smartphone-Based Ischemic Heart Disease (heart attack) Risk Prediction Using Clinical Data Mining Approaches, A Prototype Design. $19^{\text {th }}$ International Conference on Computer and Information Technology (ICCIT), IEEE,2016; 299-303.

13. Prittopaul P, Sathya S, Jayasree K. Cyber-Physical System Approach For Heart Attack Detection And Control Using Wireless 
Monitoring And Actuation System. IEEE 9th International Conference on Intelligent Systems and Control (ISCO) 2015.

14. Pawar PA. Heart Rate Monitoring System Using IR Base Sensor \& Arduino Uno. Conference on IT in Business, Industry and Government (CSIBIG) 2014.

15. Das S. The Development of A Microcontroller Based Low-Cost Heart Rate Counter For Health Care Systems. Int J Engi Trends Technol 2013;4(2):207-211.

16. Ashrafuzzaman M, Huq M, Chakraborty C, Md. Khan RM, Tabassum T, Hasan R. Heart Attack Detection Using Smart Phone. Int J Technol Enhan Emerg Engi Res 2013.
17. Isaac R, Ajaynath MS. A Real-Time Heart Monitoring System Using Android Smartphone. IEEE India Conference 2012.

18. Srinivas K, Govardhan A, Rao RG. Analysis of Coronary Heart Disease and Prediction of Heart Attack Using Data Mining Techniques. 5th International Conference on Computer Science and Education, IEEE,2010; 1344-1349.

19. Landaeta RG, Casas O, Pallas-Areny R, Heart rate detection from plantar bioimpedance measurements. IEEE Transac Biomed Engi 2008;55(3):1163-1167. 